\section{$\mathbf{m} / \mathbf{S}$}

médecine/sciences 1997 ; 13 : 145-6

\title{
LES ÉTATS-UNIS D'AMÉRIQUE, UNE TERRE D'ACCUEIL POUR TOUS LES SCIENTIFIQUES DU MONDE
}

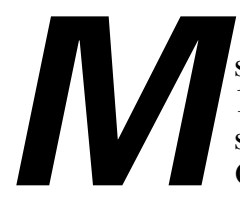
scientifique, per se, ce numéro se veut aussi de montrer que les français et autres francophones établis aux États-Unis ont, grâce à cela (?), malgré cela (?) su faire leur marque dans ce que sont médecine et sciences aujourd'hui.

Posée comme je viens de le faire, la question n'a, à mon avis, qu'une réponse sans ambiguïté.

La Seconde Guerre mondiale, le rôle majeur des États-Unis dans la victoire, la nouvelle façon de concevoir l'application de la science fondamentale qu'avaient été le Manhattan Project, le développement du radar, la fabrication industrielle de la pénicilline, l'immense pratique de la chirurgie thoracique et vasculaire au niveau des hôpitaux de campagne de l'armée et de la marine, marquèrent une génération complète (19451970) où la pré-éminence américaine était indubitable. Le rapport Flexner sur le rôle de l'État et des subventions fédérales dans le développement des sciences biologiques, la création des NIH (National Institutes of Health) et leurs subventions aux projets " extramuraux », c'est-à-dire partout où ils se justifiaient, aux États-Unis comme à l'étranger, universitaires ou non, la générosité de la Fondation Rockefeller - Rockefeller qui avait déjà accueilli Alexis Carrel, premier prix Nobel en médecine aux États-Unis en 1912, puis René Dubos - et la politique ouverte et à longue vue de ses directeurs - Warren Weaver, tout mathématicien qu'il était distribuait les premiers fonds de recherche à la «biologie moléculaire »-y compris au groupe de Lwoff à l'Institut Pasteur - Jacob, Lwoff et Monod, prix Nobel en 1965 -, la création de la médecine universitaire à plein temps avec son obligation de participer à la recherche fondamentale ou clinique, toute cette époque d'après-guerre a été incontestablement un temps où les États-Unis ont montré une nouvelle façon de fonctionner, de promouvoir la science et ses applications, en particulier en biologie et en médecine. La surprise de Spoutnik en 1957 n'a fait que renforcer les idées et concepts initiaux.

La France a mis longtemps à reconnaître la puissance constructive de cette façon de penser et d'agir, tant au niveau des individus, qu'à celui des entités à budget de l'État: la création du Cnrs, de l'INH puis de l'Inserm ont finalement réalisé pour les scientifiques français des structures qui permettaient de fonctionner d'une façon créative et où les ressources étaient distribuées en fonction de l'originalité des idées et des résultats. Le concept du jugement impartial par les pairs (the peer review system, installé dès le départ du NIH) trop longtemps ignoré ou non voulu en France, est maintenant pratique courante. 
Pendant cette génération de 1945 à 1970 dont je parlais plus haut, on venait, de par le monde, aux ÉtatsUnis, pour apprendre et travailler à leur nouvelle façon; parmi les francophones, citons : André Cournand, René Dubos - en fait arrivés avant la Seconde Guerre mondiale - ; moimême, arrivant en 1948 à McGill et l'Université de Montréal pour faire un Ph.D. en médecine expérimentale avec Hans Selye, puis allant au Baylor College of Medicine à Houston (TX, USA) de 1952 à 1970, et au Salk Institute à La Jolla (CA, USA) de 1970 à 1990 ; Baruj Benacerraf venant de Broussais à la faculté de médecine de NYU (New York University); Christian deDuve, se déplaçant de Louvain au Rockefeller Institute en un arrangement unique de double appartenance ; ajoutons aussi Gérard Debreux dans le domaine des sciences économiques. Tous, nous avons bénéficié de ce climat et de ces structures américaines que je mentionnais plus haut et tous avec succès, si l'on croit au voyage à Stockholm. Et nous avons tous laissé notre marque dans les sciences et la médecine modernes avec et par les dizaines d'élèves que nous avons formés, maintenant collègues de par le monde.

J'ai souvent pensé que nous, ici aux États-Unis, avions moins de mérite à nos succès que nos collègues de France n'en avaient pour les leurs, les conditions dans lesquelles ils travaillaient étant tellement moins simples, moins faciles.

Aujourd'hui, et depuis une quinzaine d'années, les choses ont bien changé ; le déséquilibre que je décriais ou impliquais plus haut n'existe plus ou, en tout cas, n'est plus de tout ou rien. Les collaborations sont d'égal à égal, les échanges absolument dans les deux sens, les carrières, possibles et heureuses, d'un côté ou l'autre de l'Atlantique. Et pourtant, je pense encore qu'il est plus simple - les voies sont nettes, je ne dis pas plus facile, de faire son point, de faire carrière aux États-Unis qu'il ne l'est en France. La survie des féodalités m'y étonne toujours. Peut-être, comme dans la réponse de Max Planck dans une discussion sur la difficulté à l'acceptation de la physique quantique, disparaîtront-elles une bonne fois avec tous leurs actuels tenants. Peut-être ai-je eu de la chance, je n'en ai jamais rencontré ici.

Quoi qu'il en soit de tout cela, je ne peux pas ne pas dire un mot sur la qualité de la science présentée dans ce numéro de médecine/sciences. La biologie que j'ai pratiquée en mon temps a fait place au réductionisme tout puissant de la biologie moléculaire, de ses techniques, de ses concepts. Le sommaire de $\mathrm{m} / \mathrm{s}$ pour février 1997 en est un excellent exemple. Qu'on n'oublie pas, cependant, la nécessité de la physiologie, la biologie supramoléculaire comme l'a appelée Paul Weiss, le premier a avoir utilisé molecular biology dans le sens que nous lui donnons aujourd'hui. Je pense que les comités de rédaction de médecine/sciences et ses Rédacteurs en chef en sont bien conscients. C'est ce qui fait, à mes yeux, le remarquable succès de cette revue 